\title{
Constituintes químicos do caule de Protium hebetatum (Burseraceae)
}

Túlio de Orleans Gadelha COSTA ${ }^{1}$, Richardson Alves de ALMEIDA ${ }^{1}$, Hector Henrique Ferreira KOOLEN ${ }^{1}$, Felipe Moura Araujo da SILVA ${ }^{1}$, Angelo Cunha PINTO²

\section{RESUMO}

Protium é um gênero que se destaca na família Burceraceae, compreende cerca de 146 espécies, das quais um pequeno número tem sido estudada do ponto de vista fitoquímico. Neste trabalho, foram isolados os terpenoides $\alpha$-e $\beta$-amirina, os esteroides campesterol, estigmasterol e sitosterol e a cumarina escopoletina, a partir do tronco de Protium hebetatum. As estruturas destas substâncias foram identificadas por RMN, MS, IV e por comparaçáo com dados espectrais obtidos naa literatura e com amostras autênticas.

\section{Chemical constituents from the stem of Protium hebetatum (Burseraceae)}

\section{ABSTRACT}

Protium is the largest genus in the Burceraceae family, which comprises about 146 species, of which a small number has been studied from the phytochemical point of view. In this work the terpenoids $\alpha$ - and $\beta$-amyrin, the steroids campesterol, stigmasterol and sitosterol and the coumarin scopoletin were isolated from the stem of Protium hebetatum. The structures of these substances were identified by NMR, MS, IV and comparison with spectral data from the literature and with authentic samples.

KEYWORDS: Protium, $\alpha$-amyrin, $\beta$-amyrin, campesterol, stigmasterol, sitosterol, scopoletin.

1 Departamento de Química, Universidade Federal do Amazonas, 69077-000 Manaus - AM, Brasil Email : tulio@ufam.edu.br , quimico_ufam@hotmail.com, hectorkoolen@gmail.com, felipesaquarema@bol.com.br

2 Instituto de Química da Universidade Federal do Rio de Janeiro, Ilha do Fundão - RJ, Brasil - angelo@iq.ufrj.br 


\section{INTRODUÇÃO}

Espécies de Burseraceae são endêmicas na região amazônica e possuem enormes possibilidades econômicas, destacando-se seus óleos essenciais, um dos mais importantes grupos de matérias primas para várias indústrias, notadamente as de perfumaria, alimentícia e farmacêutica (Andrade e Higuchi 2009; Marques et al. 2010). Protium é o gênero que mais se destaca dessa família com 146 espécies, ocorrendo predominantemente na Amazônia Legal e apesar desse número de espécies, poucas foram estudadas sob o ponto de vista químico (Melo et al. 2007). Desse gênero foram identificados terpenóides, esteróides, cumarinas, flavonóides e lignanas (Rüdiger et al. 2007).

As cumarinas possuem diversas propriedades farmacológicas como antiinflamatória, espasmolítica, broncodilatadora, e atua também contra o desenvolvimento de tumores, entre outras (Ito et al. 2005; Osório et al. 2004). A escopoletina, uma cumarina com atividade farmacológica comprovada, atua na regulação da pressão arterial e possui atividade bactericida contra várias espécies, incluindo Escherichia coli (Arisawa et al. 1983). A literatura registra somente duas cumarinas de Protium, sendo essas, escopoletina e propapicina (Bandeira et al. 2002; Zoghbi et al. 1981).

Este trabalho relata o estudo do caule de Protium hebetatum, de onde se obtiveram extratos para proceder com o isolamento e identificação de substâncias, por cromatografia em coluna (CC), espectros de RMN H ${ }^{1}$ e $^{13}$, UV e IV.

Nas separaçóes por CC utilizou-se sílica gel $60(0,063$ - 0,200 mm, Merck). Para cromatografias em camada delgada (CD) utilizou-se cromatofolhas Merck PL de sílica gel $60(20 \mathrm{X} 20 \mathrm{~cm}, 0,2 \mathrm{~mm}$ espessura). As revelaçōes foram efetuadas em ultravioleta (UV, $254-336 \mathrm{~mm}$ ) em equipamento HP8452A-Diode Array Spectrophotometer, com sulfato cérico e em cubas de vidro saturadas com iodo. Os solventes de corrida empregados foram das marcas Merck e grupo Química. Os pontos de fusão foram determinados em aparelhos munidos com microscópio e base Kofler. Os espectros na região do infra-vermelho (IV) foram realizados em espectrofotômetros Perkin-Elmer, Mod. 467, utilizando pastilhas de $\mathrm{KBr}$ e filmes de $\mathrm{NaCl}$. Os espectros de $\mathrm{RMN}$ $\mathrm{H}^{1}$ e $\mathrm{C}^{13}$ foram registrados em espectrômetro BRUKER mod. C200 F, utilizando $\mathrm{CDCl}_{3}$ como solvente, e referencial interno tetrametilsilano (TMS). A cromatografia em fase gasosa acoplada a espectrometria de massas (CG-MS) foi realizada em equipamento HP 5971 com sistema de dados e biblioteca Wiley/NBS.

O material botânico de Protium hebetatum (caule) foi coletado no bosque da Vivenda Verde, em zona periférica, a noroeste da cidade de Manaus - AM. Após identificado, o material foi depositado no Herbário do Instituto Nacional de
Pesquisa da Amazônia (INPA), em Manaus - AM, registrado sob o número 161.420.

Após a coleta, o caule de $P$. hebetatum foi seco a sombra e temperatura ambiente, em seguida pulverizado em um moinho, resultando em $5.800 \mathrm{~g}$ de material botânico, que foi extraído a frio em éter etílico, e álcool etílico obtendo-se 31 e $180 \mathrm{~g}$ de extrato, respectivamente.

Os extratos foram submetidos a marchas analíticas qualitativas usuais para a avaliação de seus constituintes principais, observando-se a presença de substâncias fenólicas e terpenoídicas.

O extrato etérico $(28.0 \mathrm{~g})$ foi submetido à análise em uma coluna de sílica gel, utilizando como fase móvel hexano, benzeno e acetato de etila. Após evaporação do solvente, resultaram, respectivamente, as fraçóes: EE-1 (1,3 g), EE-2 (9,4 g) e EE-3 (11,8 g).

De modo similar, o extrato alcoólico $(170 \mathrm{~g})$ foi submetido à análise em uma coluna de sílica gel, usando como eluentes: hexano, hexano/benzeno (1:1), benzeno, benzeno/ acetato de etila (1:1), acetato de etila e metanol. Após evaporaçáo dos solventes, resultaram respectivamente as fraçóes: EA-1 (1,3 g); EA-2 (2,5 g); EA-3 (2,5 g); EA-4 (7,8 g); EA-5 (28,4 g) e EA-6 (71,0 g).

As fraçôes provenientes dos extratos etérico e alcoólico foram comparadas por cromatografia em CD, observandose que as fraçóes EE-2 e EA-4 apresentaram semelhança em sua composição, e entáo, foram reunidas e codificadas como EEA-6, correspondendo a 17,2 g.

A fração de EEA-6 (16,0 g) foi cromatografada em coluna de sílica gel usando-se como fase móvel: hexano, hexano/ benzeno (7:3), benzeno, benzeno/acetato (9:1, 8:2 e 1:1), acetato, acetato/metanol (8:2), metanol. As fraçóes coletadas foram comparadas por cromatografia de $\mathrm{CD}$ e reunidas segundo a semelhança apresentada, obtendo-se 17 novas frações.

A fração EEA-6-8-13 (110 mg) apresentou-se como um sólido amarelo, que após lavado com hexano a frio, forneceu um sólido branco, codificado como amostra 01 (14,0 mg)

A fração EEA-6-16-18 $(550 \mathrm{mg})$ foi analisada através de cromatografia em CD apresentando uma mistura complexa, na qual destacou-se uma mancha de coloração lilás ao reagente sulfato cérico. Em seguida, esta fração foi cromatografada em coluna de sílica gel, utilizando como fase móvel: hexano, hexano/benzeno (9:1, 8:2 e 1:1). As fraçóes provenientes da eluição com hexano/benzeno (9:1 e 8:2) foram reunidas e após evaporaçáo do solvente apresentaram um material esbranquiçado que lavado com hexano a quente, forneceu um sólido branco de ponto de fusão $136^{\circ}-138^{\circ}$, codificado como amostra 02. 
A fração EEA-6-6-10 depois de lavada com hexano aquecido, forneceu um sólido amarelado $(18 \mathrm{mg})$, que foi codificado como amostra 03.

Após a analise desses resultados, foi possível identificar uma mistura de dois triterpenos: $\alpha$-amirina e $\beta$-amirina (Mahato et al. 1994), de três esteróides: campesterol, estigmasterol e $\beta$-sitosterol (Costa et al. 2008) e uma cumarina: escopoletina (Arisawa et al. 1983). As estruturas das substâncias isoladas foram elucidadas por métodos espectroscópicos (CG-EM, $\mathrm{CCD}, \mathrm{RMN}{ }^{1} \mathrm{H}$ e ${ }^{13} \mathrm{C}$ ), identificadas por comparaçôes na literatura e amostras autênticas.

A amostra 01 foi isolada como sólido branco com p. f. 181-184 ${ }^{\circ} \mathrm{C}$. A análise por CG/EM, apresentou dois picos com íon molecular $\mathrm{m} / \mathrm{z} 218 \mathrm{Da}$, o que foi fundamental para diagnosticar que seus dois constituintes fazem parte da ampla classe dos triterpenos, das séries $\Delta^{12}$ oleanenos e ursenos. As duas séries possuem o mesmo esqueleto carbônico diferindo somente na posiçáo de um grupo metila. Os espectros de massas desses dois isômeros são similares, com diferença apenas na intensidade dos picos. Estas observaçóes, aliadas a comparaçóes cromatográficas, dados na literatura e de amostra autêntica permitiram identificar que o amostra 01 se tratava de uma mistura constituída de $\alpha$-amirina e $\beta$-amirina (Mahato et al. 1994).

A amostra 02 foi obtida como sólido branco com p. f. 181-184 ${ }^{\circ} \mathrm{C}$. Seu espectro obtido por IV revelou absorçôes típicas de estiramento $\mathrm{OH}$ e C-H, respectivamente, em 3440 $\mathrm{cm}^{-1}$ e $2960 \mathrm{~cm}^{-1}$ e fraca absorção em $1665 \mathrm{~cm}^{-1}$, característica de estiramento $\mathrm{C}=\mathrm{C}$. Um acoplamento em $835 \mathrm{~cm}^{-1}$, correspondente à deformaçáo angular $=\mathrm{C}-\mathrm{H}$, indicou tratarse de substância alifática, confirmando a natureza esteroidal, evidenciada através de reação de Liebermann-Burchard. Contudo, o cromatograma desta amostra obtido por CG/ EM revelou 3 picos com tempos de retenção próximos, cujos espectros de massas apresentaram íons moleculares em $\mathrm{m} / \mathrm{z}$ 400, 412 e 414 Da. Por análise comparativa desses espectros, verificaram-se fragmentaçôes semelhantes, características do esqueleto esteroidal, indicando a perda da cadeia alquílica (R) lateral, com $m / z 273\left(\mathrm{C}_{19} \mathrm{H}_{29} \mathrm{O} ; \mathrm{M}-\mathrm{R}\right), \mathrm{m} / z 255\left(\mathrm{C}_{19} \mathrm{H}_{27}\right.$; $\left(\mathrm{M}-\mathrm{R}-\mathrm{H}_{2} \mathrm{O}\right), \mathrm{m} / \mathrm{z} 213\left(\mathrm{C}_{16} \mathrm{H}_{21} ; \mathrm{M}-\mathrm{R}-\mathrm{H}_{2} \mathrm{O}-\mathrm{C}_{3} \mathrm{H}_{6}\right)$, cada constituinte diferindo apenas em intensidade. Com base nestas observaçóes, inferiram-se as respectivas cadeias laterais $\mathrm{C}_{9} \mathrm{H}_{19}, \mathrm{C}_{10} \mathrm{H}_{19}, \mathrm{C}_{10} \mathrm{H}_{21}$, com o que se chegou às formulas moleculares, $\mathrm{C}_{28} \mathrm{H}_{48} \mathrm{O}, \mathrm{C}_{29} \mathrm{H}_{48} \mathrm{O}, \mathrm{C}_{29} \mathrm{H}_{50} \mathrm{O}$. Comparaçôes cromatográficas com amostra autêntica e com dados obtidos na literatura, permitiram concluir que a amostra 02 se tratava de uma mistura constituída de campesterol, estigmasterol e $\beta$-sitosterol (Costa et al. 2008).

A amostra 03, após tratamento com hexano a quente foi isolado como sólido amarelo com p. f. 203-250 ${ }^{\circ} \mathrm{C}$, seu espectro no IV indicou uma banda larga em $3.350 \mathrm{~cm}^{-1}$ característica de deformação axial do grupo O-H. Observou-se também uma absorção intensa em $1690 \mathrm{~cm}^{-1}$, em 1285 a 1140 $\mathrm{cm}^{-1}$, sugerindo a presença de uma carbonila de éster ou lactona $\alpha-\beta$ insaturada, que foi confirmada no espectro de $\mathrm{RMN}{ }^{13} \mathrm{C}$ em 161,40 ppm. A ocorrência de 3 bandas $(1650,1560$ e $\left.1510 \mathrm{~cm}^{-1}\right)$, próprias de deformaçáo axial $\mathrm{C}=\mathrm{C}$ indicaram a natureza aromática do composto, enquanto a observação de uma intensa fluorescência azul celeste em $336 \mathrm{~nm}$, aliada ao registro de observaçôes $\left(\lambda_{\text {max }}, \mathrm{CHCl}_{3}[\log \epsilon]\right)$ em $242(2,73)$, $252(2,72), 296$ (2,76), $342(3,08) \mathrm{nm}$ no espectro de UV, revelou um sistema altamente conjugado, compatível com cromóforos olefinicos, assinalados em $\delta 6,25(\mathrm{~d}, \mathrm{~J}=9,5 \mathrm{~Hz})$ e $\delta 7,58(\mathrm{~d}, \mathrm{~J}=9,5 \mathrm{~Hz})$ no espectro de RMN ${ }^{1} \mathrm{H}$ indicativos da presença de sistema $\mathrm{AB}$, característico dos prótons, $\mathrm{H}-3$ e $\mathrm{H}-4$, da ligação dupla de isomeria cis do anel pirânico. O espectro de $\mathrm{RMN}{ }^{1} \mathrm{H}$ apresentou ainda um singleto $(3 \mathrm{H}) \mathrm{em} \delta$ 3,96 configurando a presença de uma metoxila e três singletos, $\delta 6,84(1 \mathrm{H}), 6,91(1 \mathrm{H})$ e $\delta 6,17(1 \mathrm{H})$, correspondendo, respectivamente, a 2 hidrogênios do anel benzênico e ao hidrogênio da hidroxila observada no IV. Estes dados estáo de acordo com o espectro de $\mathrm{RMN}{ }^{13} \mathrm{C}$, confirmando a presença da metoxila em 56,49 ppm e de 8 carbonos $s p^{2}$ em 150,37, $149,81,144,10,143,26,113,48,111,56,107,68$ e 103,28 $\mathrm{ppm}$. Adicionalmente, o espectro de massas registrou o íon molecular em $\mathrm{m} / \mathrm{z} 192$, verificando-se uma diferença de $46 \mathrm{Da}$ em relação à cumarina $\left(\mathrm{C}_{9} \mathrm{H}_{6} \mathrm{O}_{2}\right)$, o que levou à confirmaçáo da presença dos grupos $\mathrm{OH}$ e $\mathrm{CH}_{3} \mathrm{O}$ no anel aromático e definiu a formula molecular $\mathrm{C}_{10} \mathrm{H}_{8} \mathrm{O}_{4}$ para o composto. Um fragmento intenso de $m / z 177$, correspondendo a $\mathrm{M}-\mathrm{CH}_{3}$ conduziu a localização da metoxila e hidroxila em posiçōes vizinhas (6 e 7) da estrutura cumarínica, compatível com as estruturas quinônicas e justificando os fragmentos em $\mathrm{m} / \mathrm{z}$ 149 e $121 \mathrm{Da}$. Assim como os dois singletos aromáticos do espectro de RMN ${ }^{1} \mathrm{H}$. Os fragmentos de $m / z 149(\mathrm{M}-\mathrm{C}=\mathrm{O})$, $121(\mathrm{M}-2 \mathrm{x} \mathrm{C}=\mathrm{O})$, também são coerentes com a estrutura de uma cumarina oxigenada em 6 e 7. Esses dados, comparados com os registrados na literatura e de mostra autêntica, levaram a identificação da cumarina escopoletina (Arisawa et al. 1983).

Neste trabalho, foi possível identificar e isolar uma mistura de dois terpenóides ( $\alpha$ e $\beta$-amirina), de três esteróides (campestrol, estigmasterol e sitosterol) e uma cumarina (escopoletina). Com exceção da escopoletina, todas as substâncias foram identificadas nessa espécie (Silva et al. 2009).

Sendo poucos os grupos de angiospermas realmente ricos em cumarinas, não é de se espantar que a família Burseraceae apresente um potencial de produção de cumarinas reduzido. Ainda sim, observa-se que indivíduos da ordem Sapindales ocupam uma posição de destaque na produção dessas substâncias, sendo a segunda ordem em número de ocorrências, com mais de 1700 cumarinas descritas na literatura. Com o estudo realizado, pode-se sugerir que o 
gênero Protium é constituído por espécies com potencial na produção de cumarinas.

\section{AGRADECIMENTOS}

Aos órgãos financiadores CNPq, CAPES, FAPEAM, pelo suporte financeiro às pesquisas.

\section{BIBLIOGRAFIA CITADA}

Andrade, E.A.; Higuchi, N. 2009. Produtividade de quatro espécies arbóreas de Terra Firme da Amazônia Central. Acta Amazonica, 39:105-112.

Arisawa, M.; Kinghorn, A.D.; Cordell, G.A.; Farnswort, N.R. 1983. Plant anticancer agents. XXIII. $6 \alpha$-Senecioyloxychaparrin, a new antileukemic quassinoid from Simaba multiflora. Journal of Natural Products, 46:218-221.

Bandeira, P.N.; Pessoa, O.D.L.; Trevisan, M.T.S.; Lemos, T.L.G. 2002. Metabólitos secundários de Protium heptaphyllum MARCH. Quimica Nova, 25:1078-1080.

Costa, H.N.R,; Santos, M.C.; Alcântara, A.F.C.; Silva, M.C.; França, R.C.; Veloso, D.P. 2008. Constituintes químicos e atividade antiedematogênica de Peltodon radicans (LAMIACEAE). Quimica Nova, 31:744-750

Ito, C.; Itoigawa, M.; Onoda, S.; Hosokawa, A.; Ruangrungsi, N.; Okuda, T.; Tokuda, H.; Nishino, H.; Furukawa, H. 2005. Chemical constituents of Murraya siamensis: three coumarins and their anti-tumor promoting effect. Phytochemistry, 66:567-572.
Mahato, S. B.; Kundu, A. P. $1994 .{ }^{13} \mathrm{C}$ NMR Spectra of pentacyclic triterpenoids - a compilation and some salient features. Phytochemistry, 37:1517-1575.

Marques, D.D; Sartori, R.A.; Lemos, T.L.G.; Machado, L.L; Souza, J.S.N; Monte, F.J.Q. 2010. Composiçâo química do óleo essencial de duas subespécies do Protium heptaphyllum. Acta Amazonica, 40(1): 227-2230

Melo, M.F.F.; Macedo, S.T.; Daly, D.C. 2007. Morfologia de frutos, sementes e plântulas de nove espécies de Protium Burm. f. (Burseraceae) da Amazônia Central, Brasil. Acta Botânica Brasileira, 21:503-520.

Osório, A.C.; Martins, J.L.F. 2004. Determinação de cumarina em extrato fluido e tintura de guaco por espectrofotometria derivada de primeira ordem. Revista Brasileira de Ciência Farmacêutica, 40:481-486.

Rüdiger, A.L.; Siani, A.C.; Junior, V.F.V. 2007. The chemistry and pharmacology of the South America genus Protium Burm. f. (Burseraceae). Pharmacognosy Review, 1:93-104.

Silva, J.R.A.; Zoghbi, M.G.B.; Pinto, A.C.; Godoy, R.L.O.; Amaral, A.C.F. 2009. Analysis of the hexane extracts from seven oleoresins of Protium species. Journal of Essential Oil Research, 21:305-308

Zoghbi, M.G.B.; Roque, N.F.; Gottlieb, O.R. 1981. Propacin, a coumarinolignoid from Protium opacum. Phytochemistry, 20:180.

Recebido em: 24/08/2011

Aceito em: 25/11/2011 